PROCEEDINGS OF THE

AMERICAN MATHEMATICAL SOCIETY

Volume 139, Number 5, May 2011, Pages 1681-1690

S 0002-9939(2010)10593-2

Article electronically published on September 17, 2010

\title{
SIMPLE PROOFS OF SOME RESULTS OF RESHETNYAK
}

\author{
DANIEL SPECTOR \\ (Communicated by Tatiana Toro) \\ To Pei Chen, whose love and support lift me daily
}

\begin{abstract}
In this paper we give simpler proofs of the classical continuity and lower semicontinuity theorems of Reshetnyak.
\end{abstract}

\section{Main Result}

In 1968, Reshetnyak 20 proved two important results concerning the continuity and lower semicontinuity of functionals with respect to weak-star convergence of measures. These theorems are used in a variety of areas in the calculus of variations, ranging from problems in relaxation $([1,[3,[4,[6])$ and estimates in $\Gamma$-convergence $([17,[18,[19])$ to anisotropic surface energies studied in continuum mechanics $([9,[10,[11,[14])$ and various other applications $([2,, 7,[12])$.

For $X$ a locally compact, separable metric space, let $\left[M_{b}(X)\right]^{m}$ denote the space of $\mathbb{R}^{m}$-valued measures on $X$ with finite total mass. Given $\mu \in\left[M_{b}(X)\right]^{m}$, we write $|\mu|$ for the total variation of $\mu$ and $\frac{d \mu}{d|\mu|}$ for the Radon-Nikodym derivative of $\mu$ with respect to $|\mu|$. Under these assumptions (see Proposition 1.43 and Remark 1.57 of [5), we have that $\left[M_{b}(X)\right]^{m}$ is the dual of $\left[C_{0}(X)\right]^{m}$ (the completion of the space of $\mathbb{R}^{m}$-valued continuous functions with compact support in the sup norm). Thus, for $\mu_{n}, \mu \in\left[M_{b}(X)\right]^{m}$, we have that $\mu_{n} \stackrel{*}{\rightarrow} \mu$ in $\left[M_{b}(X)\right]^{m}$ if

$$
\lim _{n \rightarrow \infty} \int_{X} \phi \cdot d \mu_{n}=\int_{X} \phi \cdot d \mu
$$

for every $\phi \in\left[C_{0}(X)\right]^{m}$. We also define another weak-star convergence, the one used in the original paper of Reshetnyak. For $\mu_{n}, \mu$ as before, we say that $\mu_{n} \stackrel{*}{\rightarrow} \mu$ in $\left(\left[C_{b}(X)\right]^{m}\right)^{\prime}$ if (1.1) holds for all $\phi \in\left[C_{b}(X)\right]^{m}$ (the space of $\mathbb{R}^{m}$-valued bounded and continuous functions equipped with the sup norm).

In 20, the following theorems are given.

Theorem 1.1. Let $X$ be a locally compact, separable metric space with measures $\mu_{n}, \mu \in\left[M_{b}(X)\right]^{m}$. Assume that $\mu_{n} \stackrel{*}{\rightarrow} \mu$ in $\left(\left[C_{b}(X)\right]^{m}\right)^{\prime}$ and that

$$
\lim _{n \rightarrow \infty} \int_{X} g\left(x, \frac{d \mu_{n}}{d\left|\mu_{n}\right|}(x)\right) d\left|\mu_{n}\right|=\int_{X} g\left(x, \frac{d \mu}{d|\mu|}(x)\right) d|\mu|
$$

Received by the editors February 16, 2010 and, in revised form, May 18, 2010.

2010 Mathematics Subject Classification. Primary 49J45; Secondary 46E27, 46G10, 28C15.

(C)2010 American Mathematical Society Reverts to public domain 28 years from publication 
for som $€^{1}$ continuous function $g: X \times \mathbb{R}^{m} \rightarrow \mathbb{R}$, positively 1-homogeneous and strictly convex in the second variable, satisfying the growth condition $|g(x, z)| \leq C|z|$ for each $(x, z) \in X \times \mathbb{R}^{m}$ and for some $C>0$. Then

$$
\lim _{n \rightarrow \infty} \int_{X} f\left(x, \frac{d \mu_{n}}{d\left|\mu_{n}\right|}(x)\right) d\left|\mu_{n}\right|=\int_{X} f\left(x, \frac{d \mu}{d|\mu|}(x)\right) d|\mu|
$$

for every continuous function $f: X \times \mathbb{R}^{m} \rightarrow \mathbb{R}$ satisfying the growth condition $|f(x, z)| \leq C_{1}|z|$ for each $(x, z) \in X \times \mathbb{R}^{m}$ and for some $C_{1}>0$.

Here, $g: \mathbb{R}^{m} \rightarrow(-\infty,+\infty]$ is positively 1 -homogeneous if

$$
g(t z)=\operatorname{tg}(z)
$$

for all $t>0$ and all $z \in \mathbb{R}^{m}$. Note that the definition for positive 1-homogeneity is not uniform throughout the literature, particularly when functions can take the value $+\infty$. We say that $g: \mathbb{R}^{m} \rightarrow(-\infty,+\infty]$ is convex if the inequality

$$
g(\theta z+(1-\theta) w) \leq \theta g(z)+(1-\theta) g(w)
$$

holds for all $\theta \in[0,1]$ and for all $z, w \in \mathbb{R}^{m}$. If $g$ is positively 1-homogeneous, this definition is equivalent to the inequality

$$
g(z+w) \leq g(z)+g(w)
$$

holding for all $z, w \in \mathbb{R}^{m}$. Following Reshetnyak 20, we say that a positively 1homogeneous function $g: \mathbb{R}^{m} \rightarrow(-\infty,+\infty]$ is strictly convex if the inequality (1.4) is strict, except when $w=0$ or $z=t w$ for some $t>0$. Note that this definition is not standard.

Theorem 1.2. Let $X$ be a locally compact, separable metric space with measures $\mu_{n}, \mu \in\left[M_{b}(X)\right]^{m}$; if $\mu_{n} \stackrel{*}{\rightarrow} \mu$ in $\left(\left[C_{b}(X)\right]^{m}\right)^{\prime}$, then

$$
\liminf _{n \rightarrow \infty} \int_{X} f\left(x, \frac{d \mu_{n}}{d\left|\mu_{n}\right|}(x)\right) d\left|\mu_{n}\right| \geq \int_{X} f\left(x, \frac{d \mu}{d|\mu|}(x)\right) d|\mu|
$$

for every continuous function $f: X \times \mathbb{R}^{m} \rightarrow \mathbb{R}$, positively 1-homogeneous and convex in the second variable, satisfying the growth condition $|f(x, z)| \leq C|z|$ for each $(x, z) \in X \times \mathbb{R}^{m}$ and for some $C>0$.

The proofs to variants of Theorems 1.1 and 1.2 have been given in [5], 19], and 20], and although the statement of the hypotheses differs, the technique is essentially the same. The idea has been to construct sequences of measures on the product space $X \times S^{m-1}$, extract a limit via compactness, and use a disintegration theorem (see [5], Theorem 2.28) to project the limiting object for analysis. Some work has been done involving arguments specific to particular problems, for example, time-dependent problems [17, as well as the desire to consider $f$ that are not necessarily 1-homogeneous ([15], [16]). However, these arguments either use the original theorem or are applicable only in a more specific context.

In this paper, we show that in the Euclidean setting it is possible to give simple proofs of Theorems 1.1 and 1.2 which do not make use of the disintegration theorem. Note that the assumption $X \subset \mathbb{R}^{N}$ is not as restrictive as it looks, since locally compact topological vector spaces are finite dimensional (see Section 1.9 in

\footnotetext{
${ }^{1}$ The English translation of this quantifier says 'for each', when in fact the original Russian says 'for some'.
} 
21])2 Moreover, the applications of Theorems 1.1 and 1.2 are generally to problems involving functions of bounded variation $[B V(\Omega)]^{m}$ (the space of functions in $\left[L^{1}(\Omega)\right]^{m}$ whose distributional derivative is an element of $\left.\left[M_{b}(\Omega)\right]^{m N}\right)$. We are now ready to state the main result.

Theorem 1.3. Let $\Omega \subset \mathbb{R}^{N}$ be open, $\mu_{n}, \mu \in\left[M_{b}(\Omega)\right]^{m}$ such that

$$
\mu_{n} \stackrel{*}{\rightarrow} \mu \text { in }\left(\left[M_{b}(\Omega)\right]^{m} \text { and }\left|\mu_{n}\right|(\Omega) \rightarrow|\mu|(\Omega) .\right.
$$

Then

$$
\lim _{n \rightarrow \infty} \int_{\Omega} f\left(x, \frac{d \mu_{n}}{d\left|\mu_{n}\right|}(x)\right) d\left|\mu_{n}\right|=\int_{\Omega} f\left(x, \frac{d \mu}{d|\mu|}(x)\right) d|\mu|
$$

for every continuous and bounded function $f: \Omega \times S^{m-1} \rightarrow \mathbb{R}$.

Note that although the hypotheses (1.5) of Theorem 1.3 seem to differ from those in Theorem 1.1 (namely, $\mu_{n} \stackrel{*}{\rightarrow} \mu$ in $\left(\left[C_{b}(\Omega)\right]^{m}\right)^{\prime}$ and $(1.2)$ ), they are in fact equivalent, as we will demonstrate following the proof of this theorem (see Remark 1.5 below). Let us begin with the following remark, which will simplify our analysis in the proofs of the theorems.

Remark 1.4. The convergence assumptions in (1.5) imply convergence in a topology stronger than the weak-star topology. As a result, if $A \subset \Omega$ is open with $\bar{A} \subset \Omega$ compact and $|\mu|(\partial A)=0$, then

$$
\lim _{n \rightarrow \infty} \int_{A} \phi \cdot d \mu_{n}=\int_{A} \phi \cdot d \mu
$$

for every $\phi \in\left[C_{b}(\Omega)\right]^{m}$, and

$$
\lim _{n \rightarrow \infty} \int_{A} \psi d\left|\mu_{n}\right|=\int_{A} \psi d|\mu|
$$

for every $\psi \in C_{b}(\Omega)$ (see [13, Corollary 1.204, Remark 1.205, and Proposition 1.206, for example).

Proof of Theorem 1.3. We claim it is enough to demonstrate

$$
\lim _{n \rightarrow \infty} \int_{\Omega^{\prime}} f\left(x, \frac{d \mu_{n}}{d\left|\mu_{n}\right|}(x)\right) d\left|\mu_{n}\right|=\int_{\Omega^{\prime}} f\left(x, \frac{d \mu}{d|\mu|}(x)\right) d|\mu|
$$

for every $\Omega^{\prime} \subset \Omega$ open with $\overline{\Omega^{\prime}} \subset \Omega$ compact and $|\mu|\left(\partial \Omega^{\prime}\right)=0$. If this is the case, we may estimate the boundary layer by

$$
\begin{gathered}
\int_{\Omega \backslash \overline{\Omega^{\prime}}} f\left(x, \frac{d \mu_{n}}{d\left|\mu_{n}\right|}(x)\right) d\left|\mu_{n}\right| \leq M\left|\mu_{n}\right|\left(\Omega \backslash \Omega^{\prime}\right), \\
\int_{\Omega \backslash \overline{\Omega^{\prime}}} f\left(x, \frac{d \mu}{d|\mu|}(x)\right) d|\mu| \leq M|\mu|\left(\Omega \backslash \Omega^{\prime}\right),
\end{gathered}
$$

where $M:=\sup _{(x, z) \in \Omega \times S^{m-1}}|f(x, z)|$. Computing the limit of (1.9), we have

$$
\begin{aligned}
\lim _{n \rightarrow \infty}\left|\mu_{n}\right|\left(\Omega \backslash \Omega^{\prime}\right) & =\lim _{n \rightarrow \infty}\left|\mu_{n}\right|(\Omega)-\lim _{n \rightarrow \infty}\left|\mu_{n}\right|\left(\Omega^{\prime}\right) \\
& =|\mu|(\Omega)-|\mu|\left(\Omega^{\prime}\right)=|\mu|\left(\Omega \backslash \Omega^{\prime}\right),
\end{aligned}
$$

\footnotetext{
${ }^{2}$ Thus, if the metric on $X$ comes from a norm or is compatible with the topology of a topological vector space, then $X$ is automatically finite dimensional.
} 
where we have used the fact that $|\mu|\left(\partial \Omega^{\prime}\right)=0$ to apply the convergence in equation (1.7) with $\psi=1$. We can then choose $\Omega^{\prime}$ appropriately to make (1.9) and (1.10) arbitrarily small.

We therefore proceed to prove (1.8). Define $\tilde{f}: \Omega \times \overline{B(0,1)} \rightarrow \mathbb{R}$ by

$$
\tilde{f}(x, z)=\left\{\begin{array}{cl}
f\left(x, \frac{z}{|z|}\right)|z| & \text { if } 0<|z| \leq 1 \\
0 & \text { if } z=0
\end{array}\right.
$$

Then since $f$ is bounded and continuous, we have that $\tilde{f}$ is bounded and continuous. Further, since $\overline{\Omega^{\prime}}$ is compact, $\tilde{f}: \Omega^{\prime} \times \overline{B(0,1)} \rightarrow \mathbb{R}$ is uniformly continuous. Thus, for every $\delta>0$, there exists a $C_{\delta}>0$ such that

$$
|\tilde{f}(x, y)-\tilde{f}(x, z)| \leq C_{\delta}|y-z|^{2}+\delta
$$

for all $x \in \Omega^{\prime}$ and for all $y, z \in \overline{B(0,1)}$. To obtain this estimate, let $\delta>0$ be given. By uniform continuity of $\tilde{f}$, there exists an $\epsilon=\epsilon(\delta)>0$ such that

$$
|\tilde{f}(x, y)-\tilde{f}(x, z)| \leq \delta
$$

for all $x \in \Omega^{\prime}$ and for all $y, z \in \overline{B(0,1)}$ with $|y-z|<\epsilon$. However, if $|y-z| \geq \epsilon$, then $\frac{|y-z|^{2}}{\epsilon^{2}} \geq 1$ so that by boundedness of $\tilde{f}$ we have

$$
|\tilde{f}(x, y)-\tilde{f}(x, z)| \leq 2 M \leq 2 M \frac{|y-z|^{2}}{\epsilon^{2}} .
$$

Combining equations (1.12) and (1.13) and defining $C_{\delta}:=\frac{2 M}{\epsilon^{2}}$ yields inequality (1.11).

Let $\varphi: \Omega \rightarrow \overline{B(0,1)} \subset \mathbb{R}^{m}$ be continuous, to be chosen later. To prove (1.8), we write

$$
\begin{aligned}
& \left|\int_{\Omega^{\prime}} f\left(x, \frac{d \mu_{n}}{d\left|\mu_{n}\right|}(x)\right) d\right| \mu_{n}\left|-\int_{\Omega^{\prime}} f\left(x, \frac{d \mu}{d|\mu|}(x)\right) d\right| \mu|| \\
& \leq\left|\int_{\Omega^{\prime}} \tilde{f}\left(x, \frac{d \mu_{n}}{d\left|\mu_{n}\right|}(x)\right) d\right| \mu_{n}\left|-\int_{\Omega^{\prime}} \tilde{f}(x, \varphi(x)) d\right| \mu_{n}|| \\
& \quad+\left|\int_{\Omega^{\prime}} \tilde{f}(x, \varphi(x)) d\right| \mu_{n}\left|-\int_{\Omega^{\prime}} \tilde{f}(x, \varphi(x)) d\right| \mu|| \\
& \quad+\left|\int_{\Omega^{\prime}} \tilde{f}(x, \varphi(x)) d\right| \mu\left|-\int_{\Omega^{\prime}} \tilde{f}\left(x, \frac{d \mu}{d|\mu|}(x)\right) d\right| \mu|| \\
& =: I+I I+I I I .
\end{aligned}
$$


We have that $I I$ goes to zero by applying the convergence result found in equation (1.7) with $A=\Omega^{\prime}$ and $\psi=\tilde{f}(x, \varphi(x))$. As for $I$ and $I I I$, by (1.11) we can bound

$$
\begin{aligned}
I+I I I \leq & \int_{\Omega^{\prime}}\left(C_{\delta}\left|\frac{d \mu_{n}}{d\left|\mu_{n}\right|}(x)-\varphi(x)\right|^{2}+\delta\right) d\left|\mu_{n}\right| \\
& +\int_{\Omega^{\prime}}\left(C_{\delta}\left|\frac{d \mu}{d|\mu|}(x)-\varphi(x)\right|^{2}+\delta\right) d|\mu| \\
\leq & \int_{\Omega^{\prime}}\left(2 C_{\delta}\left(1-\frac{d \mu_{n}}{d\left|\mu_{n}\right|}(x) \cdot \varphi(x)\right)+\delta\right) d\left|\mu_{n}\right| \\
& +\int_{\Omega^{\prime}}\left(2 C_{\delta}\left(1-\frac{d \mu}{d|\mu|}(x) \cdot \varphi(x)\right)+\delta\right) d|\mu|,
\end{aligned}
$$

where in the last inequality we have used the fact that $\frac{d \mu}{d|\mu|}(x), \frac{d \mu_{n}}{d\left|\mu_{n}\right|}(x) \in S^{m-1}$ and $|\varphi| \leq 1$. Letting $n \rightarrow \infty$, and again applying the convergence results (1.6) and (1.7), we have

$$
\begin{aligned}
I+I I I & \leq 2 \int_{\Omega}\left(2 C_{\delta}\left(1-\frac{d \mu}{d|\mu|}(x) \cdot \varphi(x)\right)+\delta\right) d|\mu| \\
& =2 \delta|\mu|(\Omega)+4 C_{\delta} \int_{\Omega}\left(1-\frac{d \mu}{d|\mu|}(x) \cdot \varphi(x)\right) d|\mu| .
\end{aligned}
$$

First choosing $\delta>0$ small, and then choosing $\varphi$ close to $\frac{d \mu}{d|\mu|}$ (since $\frac{d \mu}{d|\mu|} \in L^{1}(\Omega,|\mu|$ ), and using the density result given by Proposition 7.9 in [8), the result is demonstrated.

Remark 1.5. We can now establish the equivalence of the hypotheses of Theorem 1.1 and Theorem 1.3. That $\mu_{n} \stackrel{*}{\rightarrow} \mu$ in $\left(\left[C_{b}(\Omega)\right]^{m}\right)^{\prime}$ and (1.2) imply (1.5) is relatively straightforward, since weak-star convergence in $\left(\left[C_{b}(\Omega)\right]^{m}\right)^{\prime}$ is stronger than weak-star convergence in $\left[M_{b}(\Omega)\right]^{m}$, and applying Theorem 1.1 we conclude that (1.3) holds for $f(x, z)=|z|$. Conversely, assuming (1.5), we have that (1.2) holds for $g(x, z)=|z|$, and given $\phi \in\left[C_{b}(\Omega)\right]^{m}$, we may apply Theorem 1.3 to the function $f(x, z)=\phi(x) \cdot z$ to prove weak-star convergence in $\left(\left[C_{b}(\Omega)\right]^{m}\right)^{\prime}$.

In the remainder of the paper we give an alternative proof of Theorem 1.2 in the Euclidean setting. In view of the applications (see [3, 4, 6] ) we also study lower semicontinuity with respect to the weak-star convergence in $\left[M_{b}(\Omega)\right]^{m}$, which requires $f$ to be non-negative but allows $f$ to take the value $+\infty$. To simplify the proof, we proceed in two steps, first assuming the additional hypothesis $f(x, 0)=0$ for all $x \in \Omega$ (which is true if $f$ is real-valued by positive 1-homogeneity), and then proceeding to the general case.

Theorem 1.6. Let $\Omega \subset \mathbb{R}^{N}$ be open with measures $\mu_{n}, \mu \in\left[M_{b}(\Omega)\right]^{m}$. If $\mu_{n} \stackrel{*}{\rightarrow} \mu$ in $\left[M_{b}(\Omega)\right]^{m}$, then

$$
\liminf _{n \rightarrow \infty} \int_{\Omega} f\left(x, \frac{d \mu_{n}}{d\left|\mu_{n}\right|}(x)\right) d\left|\mu_{n}\right| \geq \int_{\Omega} f\left(x, \frac{d \mu}{d|\mu|}(x)\right) d|\mu|
$$

for every lower semicontinuous function $f: \Omega \times \mathbb{R}^{m} \rightarrow[0, \infty]$, positively 1 -homogeneous and convex in the second variable such that $f(x, 0)=0$ for all $x \in \Omega$. 
Proof. Since we have assumed $f(x, 0)=0$, we can apply Proposition 6.42 in [13] to represent $f$ as

$$
f(x, z)=\sup _{i} b_{i}(x) \cdot z,
$$

where $b_{i}: \Omega \rightarrow \mathbb{R}^{m}$ are bounded and continuous. Following the proofs of Theorem 5.14 and Theorem 6.54 in [13, without loss of generality we may assume that

$$
\liminf _{n \rightarrow \infty} \int_{\Omega} f\left(x, \frac{d \mu_{n}}{d\left|\mu_{n}\right|}\right) d\left|\mu_{n}\right|=\lim _{n \rightarrow \infty} \int_{\Omega} f\left(x, \frac{d \mu_{n}}{d\left|\mu_{n}\right|}\right) d\left|\mu_{n}\right|<\infty .
$$

Passing to a subsequence, there exists a positive Radon measure $\nu \in M_{b}(\Omega)$ such that

$$
f\left(x, \frac{d \mu_{n}}{d\left|\mu_{n}\right|}\right) d\left|\mu_{n}\right| \stackrel{*}{\rightarrow} \nu \text { in } M_{b}(\Omega)
$$

as $n \rightarrow \infty$. We claim it is enough to show that

$$
\frac{d \nu}{d|\mu|}\left(x_{0}\right) \geq f\left(x_{0}, \frac{d \mu}{d|\mu|}\left(x_{0}\right)\right) \text { for }|\mu| \text { a.e. } x_{0} \in \Omega .
$$

If we can prove (1.16), then by the Radon-Nikodym theorem we can write

$$
\nu=\frac{d \nu}{d|\mu|}|\mu|+\nu_{s}
$$

where $\nu_{s} \geq 0$ (since $f$, and in turn $\nu$, is non-negative), and we have the following inequalities:

$$
\begin{aligned}
\lim _{n \rightarrow \infty} \int_{\Omega} f\left(x, \frac{d \mu_{n}}{d\left|\mu_{n}\right|}(x)\right) d\left|\mu_{n}\right| & \geq \nu(\Omega) \geq \int_{\Omega} \frac{d \nu}{d|\mu|}(x) d|\mu| \\
& \geq \int_{\Omega} f\left(x, \frac{d \mu}{d|\mu|}(x)\right) d|\mu| .
\end{aligned}
$$

Thus, let $x_{0}$ be a Lebesgue point of $\frac{d \mu}{d|\mu|}$ with respect to the measure $|\mu|$ such that by the Besicovitch derivation theorem we have

$$
\frac{d \nu}{d|\mu|}\left(x_{0}\right)=\lim _{\epsilon \rightarrow 0} \frac{\nu\left(Q\left(x_{0}, \epsilon\right)\right)}{|\mu|\left(Q\left(x_{0}, \epsilon\right)\right)}<\infty,
$$

where $Q\left(x_{0}, \epsilon\right)$ is the cube centered at $x_{0}$ with side length $\epsilon$. Choosing a sequence of $\epsilon_{k} \rightarrow 0^{+}$such that $\nu\left(\partial Q\left(x_{0}, \epsilon_{k}\right)\right)=0$ and $|\mu|\left(2 Q\left(x_{0}, \epsilon_{k}\right)\right)=0$, by (1.6) and (1.14) we have that

$$
\begin{aligned}
\frac{d \nu}{d|\mu|}\left(x_{0}\right) & =\lim _{k \rightarrow \infty} \frac{\nu\left(Q\left(x_{0}, \epsilon_{k}\right)\right)}{|\mu|\left(Q\left(x_{0}, \epsilon_{k}\right)\right)} \\
& =\lim _{k \rightarrow \infty} \lim _{n \rightarrow \infty} \frac{1}{|\mu|\left(Q\left(x_{0}, \epsilon_{k}\right)\right)} \int_{Q\left(x_{0}, \epsilon_{k}\right)} f\left(x, \frac{d \mu_{n}}{d\left|\mu_{n}\right|}(x)\right) d\left|\mu_{n}\right| \\
& \geq \liminf _{k \rightarrow \infty} \liminf _{n \rightarrow \infty} \frac{1}{|\mu|\left(Q\left(x_{0}, \epsilon_{k}\right)\right)} \int_{Q\left(x_{0}, \epsilon_{k}\right)} b_{i}(x) \cdot \frac{d \mu_{n}}{d\left|\mu_{n}\right|}(x) d\left|\mu_{n}\right| \\
& =\liminf _{k \rightarrow \infty} \frac{1}{|\mu|\left(Q\left(x_{0}, \epsilon_{k}\right)\right)} \int_{Q\left(x_{0}, \epsilon_{k}\right)} b_{i}(x) \cdot \frac{d \mu}{d|\mu|}(x) d|\mu|,
\end{aligned}
$$


where we have used the weak-star convergence $\mu_{n} \stackrel{*}{\rightarrow} \mu$ in $\left[M_{b}(\Omega)\right]^{m}$. By the continuity of $b_{i}$, for every $\eta>0$ we have that

$$
\frac{1}{|\mu|\left(Q\left(x_{0}, \epsilon_{k}\right)\right)} \int_{Q\left(x_{0}, \epsilon_{k}\right)}\left|b_{i}(x)-b_{i}\left(x_{0}\right)\right| d|\mu| \leq \eta
$$

whenever $k$ is sufficiently large. Thus, we have that

$$
\begin{aligned}
\lim _{k \rightarrow \infty} \frac{1}{|\mu|\left(Q\left(x_{0}, \epsilon_{k}\right)\right)} \int_{Q\left(x_{0}, \epsilon_{k}\right)} & b_{i}(x) \cdot \frac{d \mu}{d|\mu|}(x) d|\mu| \\
& =\lim _{k \rightarrow \infty} \frac{1}{|\mu|\left(Q\left(x_{0}, \epsilon_{k}\right)\right)} \int_{Q\left(x_{0}, \epsilon_{k}\right)} b_{i}\left(x_{0}\right) \cdot \frac{d \mu}{d|\mu|}(x) d|\mu| \\
& =b_{i}\left(x_{0}\right) \cdot \frac{d \mu}{d|\mu|}\left(x_{0}\right)
\end{aligned}
$$

and combining this with the above, we have

$$
\frac{d \nu}{d|\mu|}\left(x_{0}\right) \geq b_{i}\left(x_{0}\right) \cdot \frac{d \mu}{d|\mu|}\left(x_{0}\right) \text {. }
$$

Finally, taking the supremum over $i$ and using equation (1.14), we obtain the inequality (1.16), and the result is demonstrated.

We now remove the hypothesis that $f(x, 0)=0$ for all $x \in \Omega$, with some subtle analysis of the set of $x \in \Omega$ such that $f(x, 0)=0$.

Theorem 1.7. Let $\Omega \subset \mathbb{R}^{N}$ be open with measures $\mu_{n}, \mu \in\left[M_{b}(\Omega)\right]^{m}$. If $\mu_{n} \stackrel{*}{\rightarrow} \mu$ in $\left[M_{b}(\Omega)\right]^{m}$, then

$$
\liminf _{n \rightarrow \infty} \int_{\Omega} f\left(x, \frac{d \mu_{n}}{d\left|\mu_{n}\right|}(x)\right) d\left|\mu_{n}\right| \geq \int_{\Omega} f\left(x, \frac{d \mu}{d|\mu|}(x)\right) d|\mu|
$$

for every lower semicontinuous function $f: \Omega \times \mathbb{R}^{m} \rightarrow[0, \infty]$, positively 1-homogeneous and convex in the second variable.

Moreover, if we assume that $\mu_{n} \stackrel{*}{\rightarrow} \mu$ in $\left(\left[C_{b}(\Omega)\right]^{m}\right)^{\prime}$, then (1.18) holds for every lower semicontinuous function $f: \Omega \times \mathbb{R}^{m} \rightarrow(-\infty, \infty]$, positively 1-homogeneous and convex in the second variable such that

$$
f(x, z) \geq b(x) \cdot z
$$

for some $b \in\left[C_{b}(\Omega)\right]^{m}$.

Proof. Define the set

$$
C:=\{x \in \Omega: f(x, 0)=0\},
$$

and note that by lower semicontinuity of $f, C$ is a closed set. We will show that without loss of generality, the complement of $C$ is $|\mu|,\left|\mu_{n}\right|$ negligible (for $n$ large), which combined with a representation for $f$ on $C$ similar to the one used in the proof of Theorem 1.6 will yield the result. To see this, note that assumption (1.15) implies that for $n$ large, say $n \geq n_{0}$,

$$
f\left(x, \frac{d \mu_{n}}{d\left|\mu_{n}\right|}(x)\right)<\infty \text { for }\left|\mu_{n}\right| \text { a.e. } x \in \Omega .
$$

Fix $n \geq n_{0}$ and let $x \in \Omega$ be such that $f\left(x, \frac{d \mu_{n}}{d\left|\mu_{n}\right|}(x)\right)<\infty$. Applying positive 1-homogeneity and using lower semicontinuity of $f$, we have that

$$
0 \leq f(x, 0) \leq \lim _{t \rightarrow 0^{+}} f\left(x, t \frac{d \mu_{n}}{d\left|\mu_{n}\right|}(x)\right)=\lim _{t \rightarrow 0^{+}} t f\left(x, \frac{d \mu_{n}}{d\left|\mu_{n}\right|}(x)\right)=0 .
$$


Thus,

$$
f(x, 0)=0 \text { for }\left|\mu_{n}\right| \text { a.e. } x \in \Omega,
$$

which combined with the weak-star convergence $\mu_{n} \stackrel{*}{\rightarrow} \mu$ in $\left[M_{b}(\Omega)\right]^{m}$ implies that

$$
0 \leq \int_{\Omega} f(x, 0) d|\mu| \leq \liminf _{n \rightarrow \infty} \int_{\Omega} f(x, 0) d\left|\mu_{n}\right|=0,
$$

so

$$
f(x, 0)=0 \text { for }|\mu| \text { a.e. } x \in \Omega \text {. }
$$

By Proposition 6.42 in [13, we may represent $f: C \times \mathbb{R}^{m} \rightarrow[0, \infty]$ as

$$
f(x, z)=\sup _{i} b_{i}(x) \cdot z,
$$

where $b_{i}: C \rightarrow \mathbb{R}^{m}$ are bounded and continuous. Now, since $C$ is closed, by the Tietze extension theorem (see [8], Theorem 4.16) we may extend $b_{i}$ to $\tilde{b}_{i}: \Omega \rightarrow \mathbb{R}^{m}$ such that $\tilde{b}_{i}$ are still bounded and continuous. But then examining the blow-up argument in the previous proof under these modifications, for any $x_{0} \in C$ and $n$ large we have

$$
\begin{aligned}
\frac{d \nu}{d|\mu|}\left(x_{0}\right) & =\lim _{k \rightarrow \infty} \frac{\nu\left(Q\left(x_{0}, \epsilon_{k}\right)\right)}{|\mu|\left(Q\left(x_{0}, \epsilon_{k}\right)\right)} \\
& =\lim _{k \rightarrow \infty} \lim _{n \rightarrow \infty} \frac{1}{|\mu|\left(Q\left(x_{0}, \epsilon_{k}\right)\right)} \int_{Q\left(x_{0}, \epsilon_{k}\right) \cap C} f\left(x, \frac{d \mu_{n}}{d\left|\mu_{n}\right|}(x)\right) d\left|\mu_{n}\right| \\
& \geq \liminf _{k \rightarrow \infty} \liminf _{n \rightarrow \infty} \frac{1}{|\mu|\left(Q\left(x_{0}, \epsilon_{k}\right)\right)} \int_{Q\left(x_{0}, \epsilon_{k}\right) \cap C} \tilde{b}_{i}(x) \cdot \frac{d \mu_{n}}{d\left|\mu_{n}\right|}(x) d\left|\mu_{n}\right| \\
& =\liminf _{k \rightarrow \infty} \liminf _{n \rightarrow \infty} \frac{1}{|\mu|\left(Q\left(x_{0}, \epsilon_{k}\right)\right)} \int_{Q\left(x_{0}, \epsilon_{k}\right)} \tilde{b}_{i}(x) \cdot \frac{d \mu_{n}}{d\left|\mu_{n}\right|}(x) d\left|\mu_{n}\right| \\
& =\liminf _{k \rightarrow \infty} \frac{1}{|\mu|\left(Q\left(x_{0}, \epsilon_{k}\right)\right)} \int_{Q\left(x_{0}, \epsilon_{k}\right)} \tilde{b}_{i}(x) \cdot \frac{d \mu}{d|\mu|}(x) d|\mu|,
\end{aligned}
$$

where we have used twice the $\left|\mu_{n}\right|$ negligibility of the complement of $C$ for $n$ large. However, this again says that

$$
\frac{d \nu}{d|\mu|}\left(x_{0}\right) \geq \tilde{b}_{i}\left(x_{0}\right) \cdot \frac{d \mu}{d|\mu|}\left(x_{0}\right)=b_{i}\left(x_{0}\right) \cdot \frac{d \mu}{d|\mu|}\left(x_{0}\right)
$$

since $x_{0} \in C$ and $\tilde{b}_{i}$ is an extension of $b_{i}$. This inequality is similar to (1.17) in Theorem [1.6] and we follow the remainder of the argument of Theorem [1.6] along with the $|\mu|$ negligibility of the complement of $C$ to reach the desired conclusion.

To prove the last claim of the theorem, assume that $\mu_{n} \stackrel{*}{\rightarrow} \mu$ in $\left(\left[C_{b}(\Omega)\right]^{m}\right)^{\prime}$ and let $f$ be as in the final part of the statement. Consider the function

$$
g(x, z):=f(x, z)-b(x) \cdot z \geq 0 .
$$


Applying the first part of the proof to the function $g$, we have

$$
\begin{aligned}
\liminf _{n \rightarrow \infty} & \int_{\Omega} f\left(x, \frac{d \mu_{n}}{d\left|\mu_{n}\right|}(x)\right) d\left|\mu_{n}\right|-\int_{\Omega} b(x) \cdot \frac{d \mu}{d|\mu|}(x) d|\mu| \\
& =\liminf _{n \rightarrow \infty} \int_{\Omega} g\left(x, \frac{d \mu_{n}}{d\left|\mu_{n}\right|}(x)\right) d\left|\mu_{n}\right| \\
& \geq \int_{\Omega} g\left(x, \frac{d \mu}{d|\mu|}(x)\right) d|\mu| \\
& =\int_{\Omega} f\left(x, \frac{d \mu}{d|\mu|}(x)\right) d|\mu|-\int_{\Omega} b(x) \cdot \frac{d \mu}{d|\mu|}(x) d|\mu| .
\end{aligned}
$$

This concludes the proof.

Remark 1.8. Under the hypotheses of Theorem 1.2, we have that (1.19) holds. We obtain this using Proposition 6.42 in [13] to conclude that for $f$ real-valued, we have

$$
f(x, z) \geq b(x) \cdot z
$$

for some $b \in[C(\Omega)]^{m}$. To show that $b \in\left[C_{b}(\Omega)\right]^{m}$, we combine this lower bound with the upper bound $f(x, z) \leq C|z|$ at $z=b(x)$. Then we have

$$
|b(x)|^{2} \leq f(x, b(x)) \leq C|b(x)|
$$

which implies that $|b(x)| \leq C$.

\section{ACKNOWLEDGMENTS}

The author thanks I. Fonseca, G. Leoni, and P. Piovano for their insightful discussions, which began the search for another proof, as well as comments and simplifications. He also thanks S. J. Spector for his helpful comments on a previous draft of this manuscript. This work is part of a thesis to be submitted by the author in partial fulfillment of the requirements for the degree of Doctor of Philosophy in Mathematics under the direction of G. Leoni at Carnegie Mellon University. The author thanks the Center for Nonlinear Analysis (NSF Grants No. DMS-0405343 and DMS-0635983) for its support during the preparation of this paper.

\section{REFERENCES}

1. E. Acerbi, G. Dal Maso, New lower semicontinuity results for polyconvex integrals, Calc. Var. Partial Differential Equations 2 (1994), 329-371. MR.1385074 (97f:49011)

2. G. Alberti, L. Ambrosio, A geometrical approach to monotone functions in $R^{n}$, Math. Z. 230 (1999), 259-316. MR1676726 (2000d:49033)

3. L. Ambrosio, G. Dal Maso, On the relaxation in $B V\left(\Omega ; R^{m}\right)$ of quasi-convex integrals, J. Funct. Anal. 109 (1992), 76-97. MR.1183605 (93j:49012)

4. L. Ambrosio, S. Mortola, V.M. Tortorelli, Functionals with linear growth defined on vector valued BV functions, J. Math. Pures Appl. (9) 70 (1991), 269-323. MR.1113814 (92j:49004)

5. L. Ambrosio, N. Fusco, D. Pallara, Functions of bounded variation and free discontinuity problems, Oxford Mathematical Monographs, The Clarendon Press, Oxford University Press, New York, 2000. MR1857292 (2003a:49002)

6. G. Bellettini, G. Bouchitté, I. Fragalà, $B V$ functions with respect to a measure and relaxation of metric integral functionals, J. Convex Anal. 6 (1999), 349-366. MR1736243 (2000k:49016)

7. G. Buttazzo, P. Guasoni, Shape optimization problems over classes of convex domains, J. Convex Anal. 4 (1997), 343-351. MR1613491 (99b:49039)

8. G. Folland, Real Analysis: Modern Techniques and Their Applications. John Wiley and Sons, New York, 1999. MR1681462 (2000c:00001) 
9. I. Fonseca, Lower semicontinuity of surface energies, Proc. Roy. Soc. Edinburgh Sect. A 120 (1992), 99-115. MR1149987 (94j:73069)

10. I. Fonseca, The Wulff theorem revisited, Proc. Roy. Soc. London Ser. A 432 (1991), 125-145. MR.1116536 (92e:49053)

11. I. Fonseca, S. Müller, A uniqueness proof for the Wulff theorem, Proc. Roy. Soc. Edinburgh Sect. A 119 (1991), 125-136. MR1130601 (93c:49026)

12. I. Fonseca, G. Leoni, J. Malý, Weak continuity and lower semicontinuity results for determinants, Arch. Ration. Mech. Anal. 178 (2005), 411-448. MR2196498 (2006m:49020)

13. I. Fonseca, G. Leoni, Modern methods in the calculus of variations: $L^{p}$ spaces, Springer Monographs in Mathematics, Springer, New York, 2007. MR2341508 (2008m:49001)

14. C. Herring, Some Theorems on the Free Energies of Crystal Surfaces, Phys. Rev. 82 (1951), 87-93.

15. J. Kristensen, F. Rindler, Relaxation of signed integral functionals in BV, Calc. Var. 37 (2010), 29-62. MR2564396

16. J. Kristensen, F. Rindler, Characterization of Generalized Gradient Young Measures Generated by Sequences in $W^{1,1}$ and BV, Arch. Rational Mech. Anal., 197, no. 2 (2010), 539-598.

17. R. Kohn, F. Otto, M.G. Reznikoff, E. Vanden-Eijnden, Action minimization and sharpinterface limits for the stochastic Allen-Cahn equation, Comm. Pure Appl. Math. 60 (2007), 393-438. MR2284215(2008a:35053)

18. N.Q. Le, A gamma-convergence approach to the Cahn-Hilliard equation, Calc. Var. Partial Differential Equations 32 (2008), 499-522. MR2402921 (2009d:35154)

19. S. Luckhaus, L. Modica, The Gibbs-Thompson relation within the gradient theory of phase transitions, Arch. Rational Mech. Anal. 107 (1989), 71-83. MR1000224 (90k:49041)

20. Y.G. Reshetnyak, Weak convergence of completely additive vector funtions on a set (Russian) Sibirski Mat. Zh. 9 (1968) 1386-1394; translation in Siberian Math. J. 9 (1968), 1039-1045. MR.0240274 (39:1623)

21. W. Rudin, Functional Analysis, International Series in Pure and Applied Mathematics, McGraw-Hill, Inc., New York, 1993. MR.1157815 (92k:46001)

Department of Mathematics, Carnegie Mellon University, Pittsburgh, Pennsylvania 15213-3890

E-mail address: dspector@andrew.cmu.edu 